\title{
Neglected congenital adrenal hyperplasia presenting as recurrent abdominal pain
}

\begin{abstract}
Congenital adrenal hyperplasia $(\mathrm{CAH})$ is an autosomal recessive disorder with the incidence of the classic type being 1:15,000 births worldwide. It results from defective synthesis of steroid hormones because of deficiency of one of the five enzymes required for synthesis of cortisol. Cases of severe forms of $\mathrm{CAH}$ are often missed in developing countries where there is no program for newborn screening with many of them dying in neonatal periods. Management of the mild forms are also hindered or delayed because of superstitions, ignorance and poverty. We present an 11years old girl who presented with abnormal genital since birth and recurrent monthly lower abdominal pain of eight month duration. She had Tanner stage 4 breasts, normal female pubic hair distribution, clitoromegaly, fused scrotalized labia with urogenital sinus and absent vaginal opening. Abdominal ultrasound showed normal female reproductive organs; she had advanced bone age of 16years and elevated serum 17-hydroxyprogesterone level. She's being worked up for possible genitoplasty.
\end{abstract}

Volume 8 Issue 2 - 2020

\author{
Oluwayemi IO,' Oyebanji $\mathrm{AH},{ }^{2}$ Omowaye \\ $\mathrm{MT}^{2}$ \\ 'Department of Paediatrics, Ekiti State University, Ado-Ekiti, \\ Nigeria \\ 2Department of Paediatrics, ABUAD Multi System Hospital, \\ Ado-Ekiti, Nigeria
}

Correspondence: Isaac Oludare Oluwayemi, Department of Paediatrics, Faculty of Clinical Sciences, College of Medicine, Ekiti State University,Ado-Ekiti, Ekiti State, Nigeria, Tel +2348034052536, Email isaac.oluwayemi@eksu.edu.ng

Received: March 27, 2020 | Published: April I3, 2020

Keywords: Congenital adrenal hyperplasia, neglected, abdominal pain, hematocolpos, urogenital sinus

\section{Introduction}

Defective synthesis of steroid hormones from the adrenal cortex is responsible for the different array of clinical features in congenital adrenal hyperplasia (CAH). In more than $90 \%$ of cases $\mathrm{CAH}$ is caused by a defect in the 21-hydroxylase gene (CYP 21). ${ }^{1,2}$ The enzyme, 21-hydroxylase, catalyses the conversion of 17-hydroxyprogesterone to 11-deoxycorticol, a precursor of aldosterone. Because of this loss of enzyme function, 21-hydroxylase deficient patients cannot synthesize cortisol efficiently with consequent overproduction of cortisol precursors by the adrenal cortex when stimulated by corticotrophins. ${ }^{1-3}$ Some of the cortisol precursors are diverted to the biosynthesis of sex hormone which is responsible for the clinical features of androgen excess, like clitoral enlargement and rapid growth, seen in affected children. ${ }^{1-3} \mathrm{CAH}$ is an autosomal recessive disorder with the classic (severe) form having an incidence of 1:15,000births worldwide while the non classic (mild) form occurs in 1:1000births worldwide and is more common (up to 1:20) in certain ethnic groups. ${ }^{4}$

\section{Case presentation}

We present F.O. an 11year old female adolescent who presented with abnormal genitals since birth and recurrent lower abdominal pain of 8month duration. Abnormal genitalia were noticed at birth, described as "no vagina" with an enlarged clitoris. Parents were told to take child to a tertiary teaching hospital for management but refused to go because of financial constraint. Patient appeared to be doing well and actually grew taller than her peers between the ages of 3 and 8years. She however developed recurrent lower abdominal pain 8 months before presentation, occurring monthly and lasting for about 4-5days associated with nausea and vomiting. No history of abdominal swelling. She started developing breast buds about 4 years prior to presentation and both breasts are well developed but yet to experience monthly vaginal bleeding since she has no vagina opening. She urinates through an opening (urogenital sinus) located at the anterior $1 / 4^{\text {th }}$ of the fused scrotalized labia. She had normally situated anal opening.

She's the first of two children to her mother in a polygamous setting. Mother is late and father has other children with no similar history in any family member. There is no history of consanguinity and no previous history of surgery. Examination revealed a female adolescent, not acutely ill looking, with a normal weight of $47.2 \mathrm{~kg}$ $\left(75^{\text {th }}-90^{\text {th }}\right.$ percentile $)$ and a normal height of $1.5 \mathrm{~m}$ ( $75^{\text {th }}$ percentile $)$. She had a normal blood pressure of $110 / 58 \mathrm{mmHg}\left(50^{\text {th }}\right.$ percentile for age and sex) and normal heart sounds. Breasts were tanner stage 4, had normal female pubic hair distribution (Tanner stage 4). Abnormal urogenital system (Figure 1) showing clitoromegaly, patent urethra opening (urogenital sinus), but fused scrotalized labial folds.

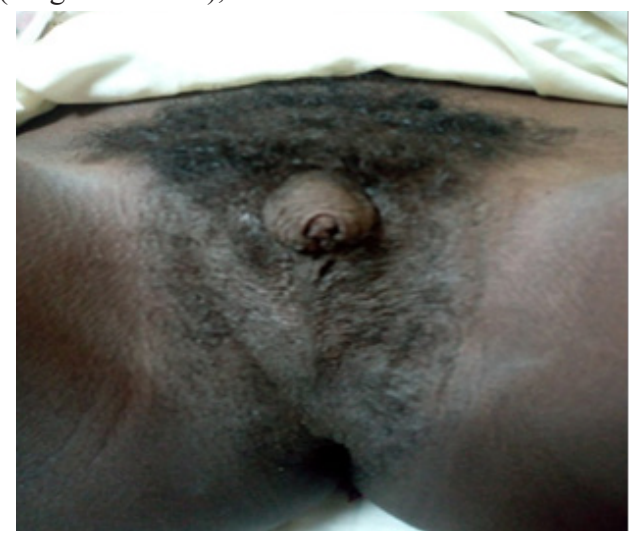

Figure I Clitoromegaly with fused scrotralized fused labia in $\mathrm{CAH}$.

An assessment of Congenital adrenal hyperplasia with haematocolpos and urogenital sinus was made. X-ray of the left hand and wrist joint for bone age shows an advanced bone age of 16years. Abodomino-pelvic ultrasonography shows normally sized pubertal ovaries and uterus. No testicular tissue seen. Serum 
17-Hydroxyprogesterone was $1.56 \mathrm{ng} / \mathrm{ml}$ (normal range for premenopausal females follicular phase $=0.32-1.47 \mathrm{ng} / \mathrm{ml})$. Patient is presently being co-managed with the Gynaecologists and Plastic surgeons who are working patient up for possible vaginoplasty.

\section{Discussion}

The index patient, an adolescent, was obviously psychologically disturbed by her abnormal genital described by her as "absent vagina". CAH patients who had early medical and surgical intervention have been reported to be well adjusted psychologically comparable to normal population..$^{5-7}$ This could have been prevented in this patient if she has been taken for medical and surgical intervention early in life. The recurrent monthly lower abdominal pain experience by this patient is likely to be caused by hematocolpos. Hematocolpos is described as blood collection in the distal closed vagina usually diagnosed in female adolescents with no menstruation and cyclic lower abdominal pain. ${ }^{8}$ It has an incidence of 1:2000female adolescent and it is caused by imperforate hymen in $90 \%$ of cases. ${ }^{9}$ The index patient has a urethra opening in the anterior $1 / 4^{\text {th }}$ of the fused srotalized labia, a form of urogenital sinus common in virilized female $\mathrm{CAH}$ patients. ${ }^{10}$ The surgical management of urogenital sinus is the most challenging part of genitoplasty in virilized CAH patients. The separation of the urinary and genital tract is particularly difficult when urogenital sinus is long with high vaginal confluence but less cumbersome when the urogenital sinus is short with low vaginal confluence. ${ }^{11-13}$ Urogenital sinus may persist or recur with likely trauma to the urethra and vagina if the separation failed. ${ }^{14}$ Early identification, diagnosis and treatment of CAH patients will reduce virilization and attendant complications. Paediatricians and General practitioners should also make an habit of examining the genitalia of adolescents presenting with recurrent lower abdominal pain to look for uncommon causes as in the index patient.

\section{Conclusion}

Congenital adrenal hyperplasia occurs in our environment. Genitalia of newborn must be routinely examined and those with any abnormality should be referred early for specialist care to prevent avoidable psychological trauma and costly surgical interventions.

\section{Acknowledgments}

We wish to appreciate the management and staff of Afe Babalola Multisystem Hospital, Ado-Ekiti, Nigeria for providing the enabling environment for the diagnosis and management of the patient.

\section{Conflicts of interest}

The authors declare that there is no conflict of interest.

\section{Funding}

None.

\section{References}

1. White PC, Speiser PW. Congenital adrenal hyperplasia due to $21-$ hydroxylase deficiency. Endocrine Reviews. 2000;21(3):245-291.

2. New MI. An update of congenital adrenal hyperplasia. Annals of the New York Academy of Sciences. 2004;1038:14-43.

3. Speiser PW, White PC. Congenital adrenal hyperplasia. New England Journal of Medicine. 2003;349:776-788.

4. Merke D, Kabbani M. Congenital adrenal hyperplasia: epidemiology, management and practical drug treatment. Paediatric Drugs. 2001;3(8):599-611.

5. Idris AN, Chandran V, Zakaria SZS, et al. Behavioural Outcome in Children with Congenital Adrenal Hyperplasia: Experience of a Single Centre. International Journal of Endocrinology. 2014.

6. Berenbaum SA, Bryk KK, Duck SC, et al. Psychological adjustment in children and adults with congenital adrenal hyperplasia. Journal of Pediatrics. 2004;144(6):741-746.

7. Berenbaum SA, Bryk KK, Duck SC. Normal intelligence in female andmale patients with congenital adrenal hyperplasia. International Journal of Pediatric Endocrinology. 2010.

8. Makris GM, Macchiella G, Vaidakis D, et al. Abdominal tumor in a 14 year old Adolescent: Imperforate Hymen Resulting in Hematocolpos- A case Report and Review of Literature. Case Reports in Obstetrics and Gynecology. 2015.

9. Sakalkale R, Samarakkody U. Familial occurrence of imperforate hymen. Journal of Pediatric and Adolescent Gynecology. 2005;18(6):427-429.

10. Sircili MHP, Bachega TSS, Madureira G, et al. Surgical Treatment after Failed Primary Correction of Urogenital Sinus in Female Patients with Virilizing Congenital Adrenal Hyperplasia: Are Good Results Possible? Frontiers in Pediatrics. 2016;4:118.

11. Schnitzer JJ, Donahoe PK. Surgical treatment of congenital adrenal hyper-plasia. Endocrinol Metab Clin North Am. 2001;30(1):137-154.

12. Rink RC, Pope JC, Kropp BP, et al. Reconstruction of the high urogenital sinus: early perineal prone approach without division of the rectum. $J$ Urol. 1997;158:1293-1297.

13. Hendren WH, Atala A. Repair of the high vagina in girls with severely mas-culinized anatomy from the adrenogenital syndrome. $J$ Pediatrc Surg. 1995;30(1):91-94.

14. Crouch NS, Creighton SM. Long-term functional outcomes of female genital reconstruction in childhood. BJU Int. 2007;100(2):403-407. 